\title{
MECHANICAL VIBRATION MEASUREMENTS ON TTF CRYOMODULES
}

\author{
A. Bosotti, C. Pagani, R. Paparella, P. Pierini, D. Sertore INFN LASA, Milano, Italy \\ R. Lange, DESY, Hamburg \\ R. De Monte, M. Ferianis, ELETTRA, 34012 Trieste Italy
}

\begin{abstract}
Few of the TTF cryomodules have been equipped with Wire Position Monitors (WPM) for the on line monitoring of cold mass movements during cool-down, warm-up and operation. Each sensor can be used as a detector for mechanical vibrations of the cryostat. A Digital Receiver board is used to sample and analyze with high frequency resolution, the WPM picked up signals, looking to its amplitude modulation in the microphonic frequency range. Here we describe the new acquisition system and show the first vibration spectra detected during normal operation of the TTF cryomodules \# 4 and \#5.
\end{abstract}

\section{INTRODUCTION}

A Wire Position Monitor (WPM) system has been developed for on-line monitoring of the cold mass during cooldown and operation. The analysis data taken by the WPM system during thermal cycles contributed to the upgrades that brought to the actual generation of cryomodules. Furthermore, together with the cryomodule evolution, the WPM system sustained substantial modifications and simplifications, starting from the two chains of 18 detectors of the first generation of cryomodules, to the current one of a single chain of seven detectors (placed in critical positions: at each end, at the three posts and between the posts), screwed through a support to a stainless steel arm which is welded to the gas return pipe (GRP). In Fig. 1 the drawing of the cryomodule \# 5 is shown, together with the longitudinal monitor positions. Each WPM behaves as a microstrip directional coupler. The monitors description and all the thermal cycles monitoring results, that demonstrated that the cold mass displacements seem to be consistent with TESLA specifications, are reported elsewhere (for ex. [1]). Now four WPMs are used as vibration detectors, considering that the low frequency vibrations of the cold mass, amplitude modulate the RF signals picked up by the microstrips. In this way the microphonics (and the sub- microphonics) can be recovered de-modulating the microstrip RF signal. For this purpose the WPM control electronics has been upgraded inserting a 4 channel Digital Receiver Board, which recovers the base-band signal like a sort of AM software radio. The de-modulated signals are converted into positions via the same two dimension $3^{\text {rd }}$ order polynomial, used to convert the WPM electrical signals into cold mass displacements. Of course, only transverse vibration in the horizontal and vertical planes can be detected.

\section{SYSTEM DESCRIPTION}

The vibration detection system is a super-heterodyne receiver, where the base-band demodulation is made digitally. A block diagram of the system is shown in Fig. 2.

The system is interfaced with the DOOCS-based TTF control system via a VME board computer, and can be remotely controlled.

Vibration spectra are locally stored to the VME board computer hard disk, and can be transferred through secure FTP protocol, for data analysis. A short description of the main system components follows.

RF Source

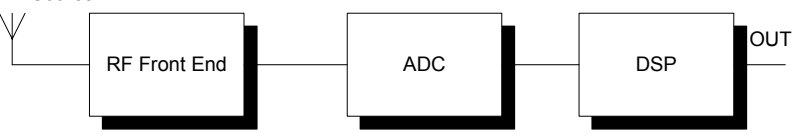

Figure 2: Digital receiver concept.

\section{RF Front End}

Two WPM readout electronics boards have been modified inserting RF buffers after their input stage, to work as the RF front end circuitry for the digital receiver. The function of the RF front end is to read the four microstrips of the desired WPM, and to down-convert the RF signal to the $10 \mathrm{MHz}$ IF signal. In Fig. 3 the RF input stage block diagram is shown. The RF signals coming

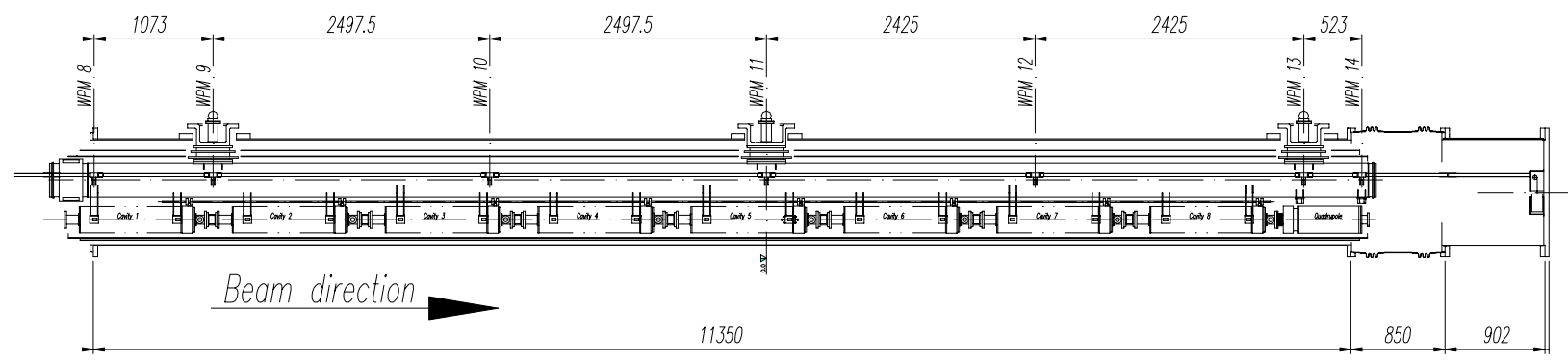

Figure 1: The longitudinal positions of the WPM sensors in cryomodule \# 5. 
from the monitor are applied to a four channel RF multiplexer, and are switched to a common read out and processing circuitry.

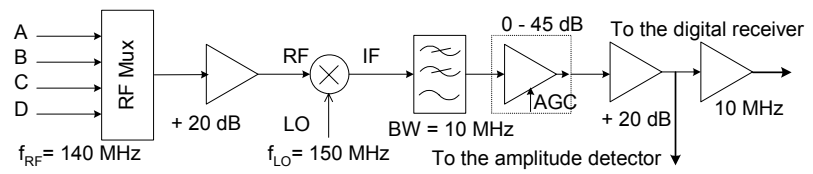

Figure 3: RF input stage.

\section{Digital Receiver}

The digital receiver board is a Quad Digital Receiver (QDR) module from Instrumentation Technologies [2]. The IF signals from the RF front end enter the QDR, where they are first filtered, and then sampled. The Analog Devices 14 bit AD9644 analog to digital converter is used for the A to D conversion. The AD9644 is mounted on a mezzanine board, attached to the main board. A block diagram of the QDR board can be found in Fig. 4.

The rest of the processing is done in a digital way. The Digital Downconverter (DDC) translates the carrier to base-band and then applies filtering and decimation, gain scaling, resampling and Cartesian to polar coordinate conversion.

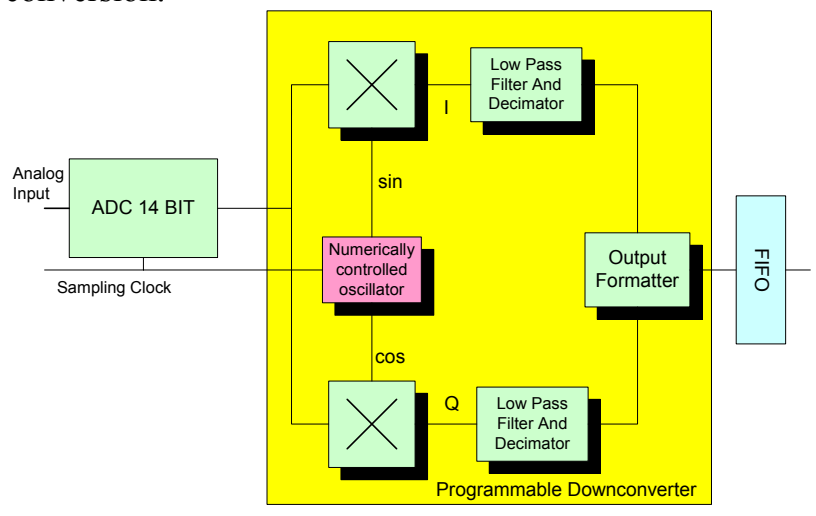

Figure 4: Digital receiver functional diagram.

\section{VIBRATION MEASUREMENTS}

Vibration measurement is a field well advanced at DESY [3] (and in all the other laboratories with big accelerators), specially in the field of ground vibrations. Results from tests using geophones, seismometers and piezos are reported in literature, as in [4] and [5]. Our effort in this field is to integrate the WPMs in the vibration sensor family. A nice feature of the WPMs is that they work in the same way at cold and warm conditions and are well distributed along the cryomodules. For this purpose, the WPMs \# 11, 12, 13, 14 , i.e. the last four monitors of the module \# 5 , has been connected to the digital receiver via the modified electronic boards. In particular, the monitor \# 11 is placed close to the central post, where we expect to find the minimum of the noise, while the monitors \# 13 and \# 14 are placed on the quadrupole. A possible limitation to the detection of the vibration spectra could be the amplitude of the stretched wire natural oscillations. The frequencies of such oscillations can be found using the vibrating string equation. We find

$$
f_{n}=\frac{n}{2 \ell} \sqrt{\frac{F}{\rho A}}=n \cdot 6.362 \mathrm{~Hz}
$$

where $\ell$ is the stretched wire length, $\mathrm{F}$ the applied tensile strength, $\rho$ the wire density and $\mathrm{A}$ its cross section.

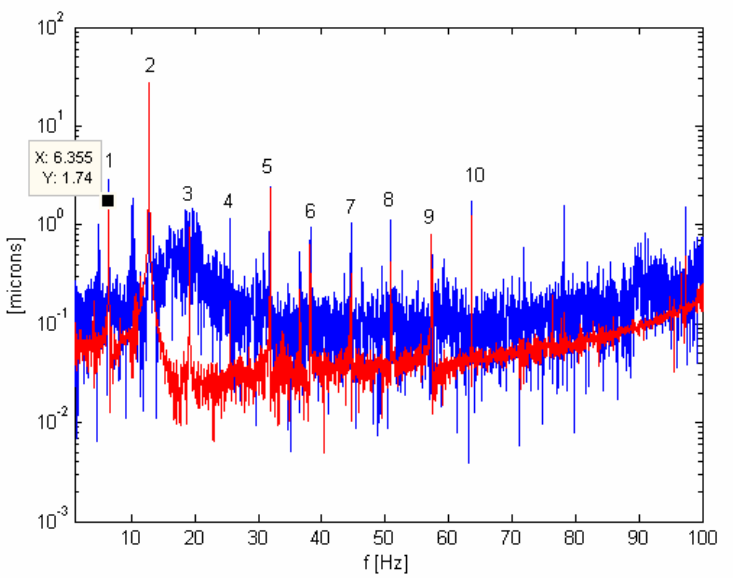

Figure 5: Wire oscillations in the horizontal plane, detected by WPM \# 11 and \# 14, superimposed to the cold mass vibration spectra. The harmonic number is reported close to each spectral line.

Looking to Fig. 5, where as an example we report the spectra of vibrations in the horizontal plane of the \# 11 (central) and \# 14 (end) detectors, we see that the wire proper vibration spectral lines (fundamental and harmonics) overcome the cold mass mechanical vibration lines (up to the $28 \mu \mathrm{m}$ of the $2^{\text {nd }}$ harmonic at the centre of the cryomodule). On the other hand, being their frequencies well predictable by equation (1), which completely agrees with the experimental data, it's easy to filter them when processing the data.

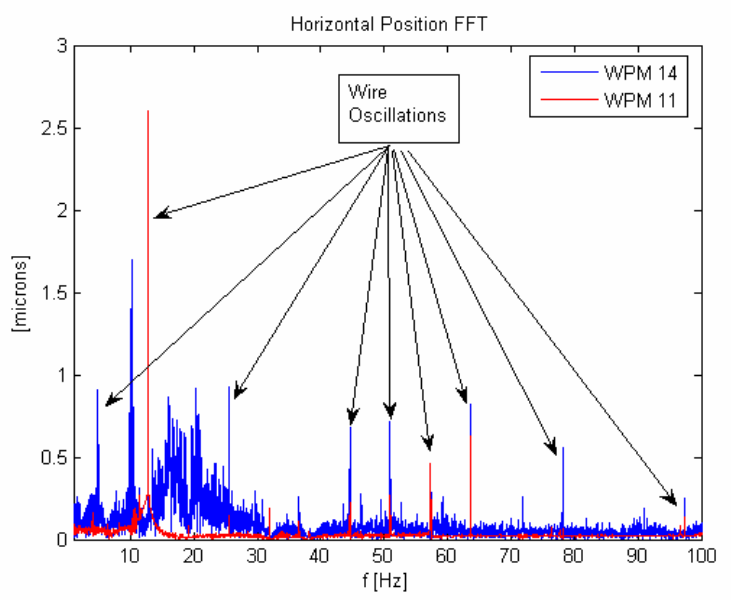

Figure 6: Cold mass vibration spectra in the horizontal plane detected by WPM \# 11 and \# 14. A residual of wire proper oscillation (after filtering) is still present. 
The filtered spectra are shown in figures 6 and 7. The data comes from $30 \mathrm{~s}$ acquisition time at $5 \mathrm{kHz}$ (nominal) sampling frequency. As expected the vibration noise is considerably low close to the central post (WPM \# 11), compared to the cryomodule end (WPM \# 14). The evidence of this comes from the analysis of the spectra shown, corresponding to vibration spectra in the horizontal and vertical planes respectively.

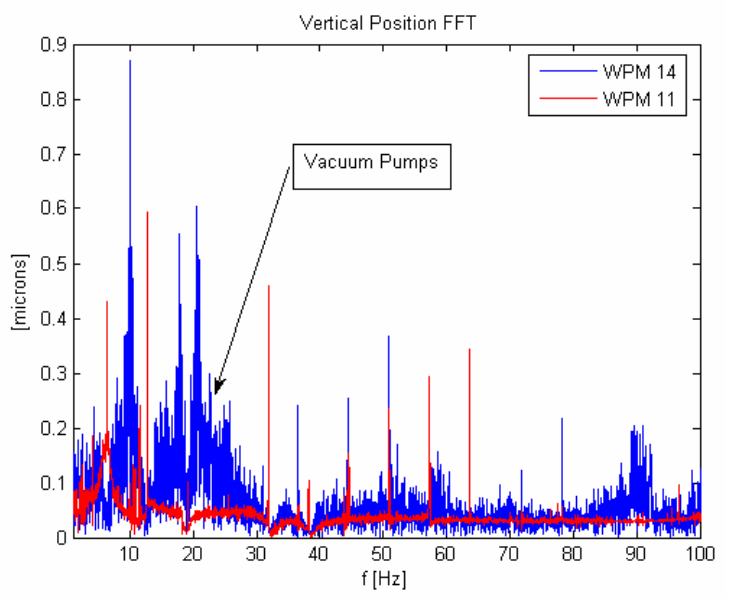

Figure 7: Cold mass vibration spectra in the vertical plane detected by WPM \# 11 and \# 14.

Looking to the blue line spectra, coming from WPM 14 , one can see that significant amount of noise is present between $10 \mathrm{~Hz}$ and $30 \mathrm{~Hz}$, due to the proximity of vacuum pumps and similar devices, and under $10 \mathrm{~Hz}$, possibly due to the cryogenic system. On the contrary, the spectra of the WPM 11 signals, which is at the central post position where all the cold mass is constrained, show only the harmonics (filtered) of the wire oscillations. The vertical scales have been intentionally left linear to make easier the vibration lines identification from the wire oscillation harmonics.

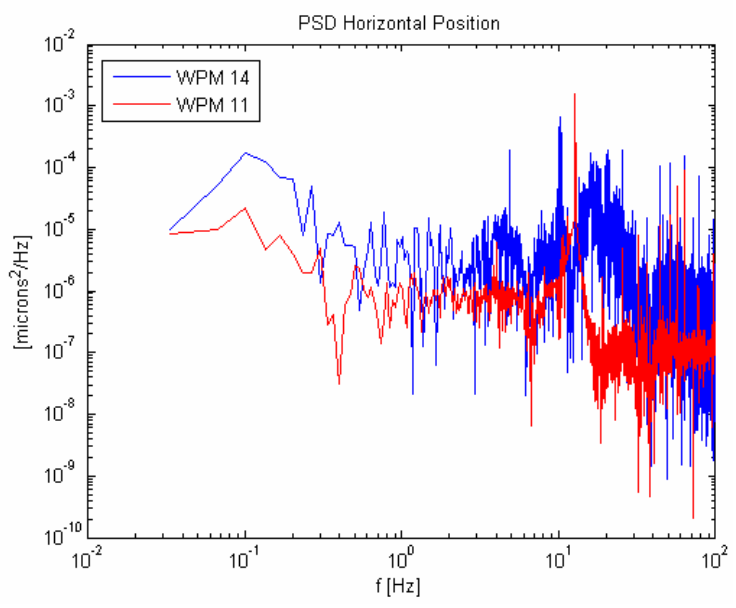

Figure 8: Power Spectral Density of the cold mass vibrations in the horizontal plane detected by WPM \# 11 and \#14.
The differences in the vibration noise is again more evident looking to the power spectral densities of the figures 8 and 9 , where if one excludes the wire oscillations, the PSDs of the signal coming from the central sensor are at least one order of magnitude lower with respect to the other at the cryomodule end.

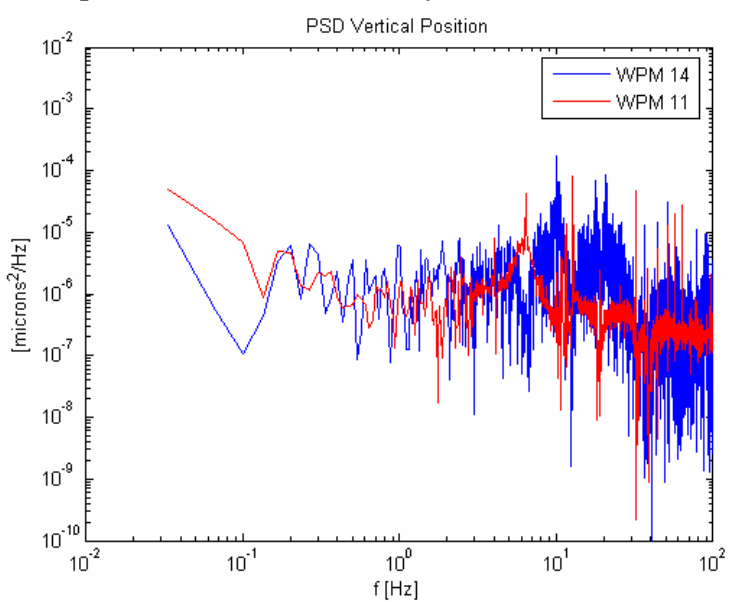

Figure 9: Power Spectral Density of the cold mass vibrations in the vertical plane detected by WPM \# 11 and $\# 14$.

\section{CONCLUSIONS}

The spectra taken with this non optimized preliminary set up, show that the WPM can be used to detect low frequency vibrations. Wire oscillations, that "dirty" the data, can be removed by filtering the spectra. Higher resolution, towards nanometres, can be achieved by properly setting the digital receiver input channel gains and electronics performance.

Further work end experiments are planned, similar to the ones reported in $[4,5]$ to explain the source of noise associated to most of the spectral lines detected.

\section{ACKNOWLEDGEMENTS}

We wish to acknowledge the support of M. Bonezzi, INFN Milano, the MKS1 group at TTF, G. Grygiel and O. Hensler from MVP group for their support. Special thanks go to Dr. G. Kreps for his infinite kindness.

\section{REFERENCES}

[1] A. Bosotti, C. Pagani, P. Pierini, R. Lange, R. De Monte, M. Ferianis, Analysis of the Cold Mass Displacements at the TTF, EPAC'04, Lucerne, July 2004, p. 1681.

[2] Instrumentation Technologies, QDR, Technical Reference User Manual, November 12, 2002.

[3] http://ground-vibrations.desy.de/index_eng.html

[4] H. Brueck, Vibration Measurements at TTF, TESLA Meeting, Hamburg March 31, 2005.

[5] W. Bialowons and H. Ehrlichmann, First Results Of The Vibration Measurements With Seismic Sensors At TTF Modules, XFEL LINAC Module Meeting Hamburg, September 2, 2004. 